\title{
MAGNTIC FIELD TRIMMING STUDIES FOR A SEPARATED-SECTOR CYCLOTRON
}

\section{E. D. Hudson, J. A. Martin, M. L. Mallory, F. E. McDaniel, and F. Irwin}

Oak Ridge National Laboratory, " Oak Ridge, Tennessee 37830, USA

\section{Abstract}

Magnetic field studies were made for a foursector, $K=330$, separated-sector cyclotron using a 1/10 scale model of a single sector equipped with 11 triming coils. Dat a are presented showing the effects of saturation at high magnetic ficlds on the field contour and on the trimming coil characteristics. Some implications of these measurements for the design of separated-sector machine magnets are given.

\section{Introduction}

In 1969 the APACHE (Accelerator for the Physics and Chemistry of lleavy Elements) project was proposed. 1) The project included a $k=330$ ( $\left.E=\mathrm{Kq}^{2} / \mathrm{A} \mathrm{MeV}\right)$ cyclotron with a wide range of capabilities. It would accelerate $\mathrm{U}^{36+}$ ions to $7.5 \mathrm{MeV} / \mathrm{amu}, \mathrm{light}$ heavy ions such as $\mathrm{C}^{6+}$ to about $80 \mathrm{MeV} / \mathrm{amu}$, and protons to an energy of $300 \mathrm{MeV}$. A $16 \mathrm{MV}$ tanden electrostatic accelerator was the injector for heavy ions; the injector for 1 ight ions could be either the tanden or a compact cyclotron. The principal characteristics of the cyclotron are given in Table 1 .

Table 1

Characteristics of Separated-Sector Cyclotron

\begin{tabular}{lll}
\hline & $\mathrm{U}^{36+}$ Ions & Protons \\
\hline
\end{tabular}

Injection charge-tomass ratio

0.15

1.0

Injection energy, Mev/amu

16.6

Final energy, MeV/amu

$\begin{array}{ll}7.5 & 300.0\end{array}$

Maximum magnetic

field, $k G$

15.0

15.5

Magnet fraction $f$

0.52

$\mathrm{B} \rho_{\max }, \mathbf{k G}-\mathrm{cm}$

Number of sectors

4

Beam radius,

Inner, $\overline{\mathbf{r}}_{i}, \mathrm{~cm}$

90.4

318.0

$\mathrm{RF}$ frequency, Mliz

Magnet weight, tons

9.45

29.34

21900

Outer diameter of cyclotron, $m$
10.7
A separated-sector magnet with four $47^{\circ}$ sectors was chosen. This configuration has a favorable ratio of average to maxinum magnetic field which reduces cyclotron size, and has good axial and radial focusing characteristics. The expected ion focusing behavior, shown in Fig. 1, is based on calculations done in cooperation with B. M. Bardin at Indiana University. The straight line is from calculations using the hard-edged approximation. The curved lines for uranıum ions and for protons are from calculations that approximate the effects of rounded edges on the azimuthal magnetic field profile of magnets of practical geometry.

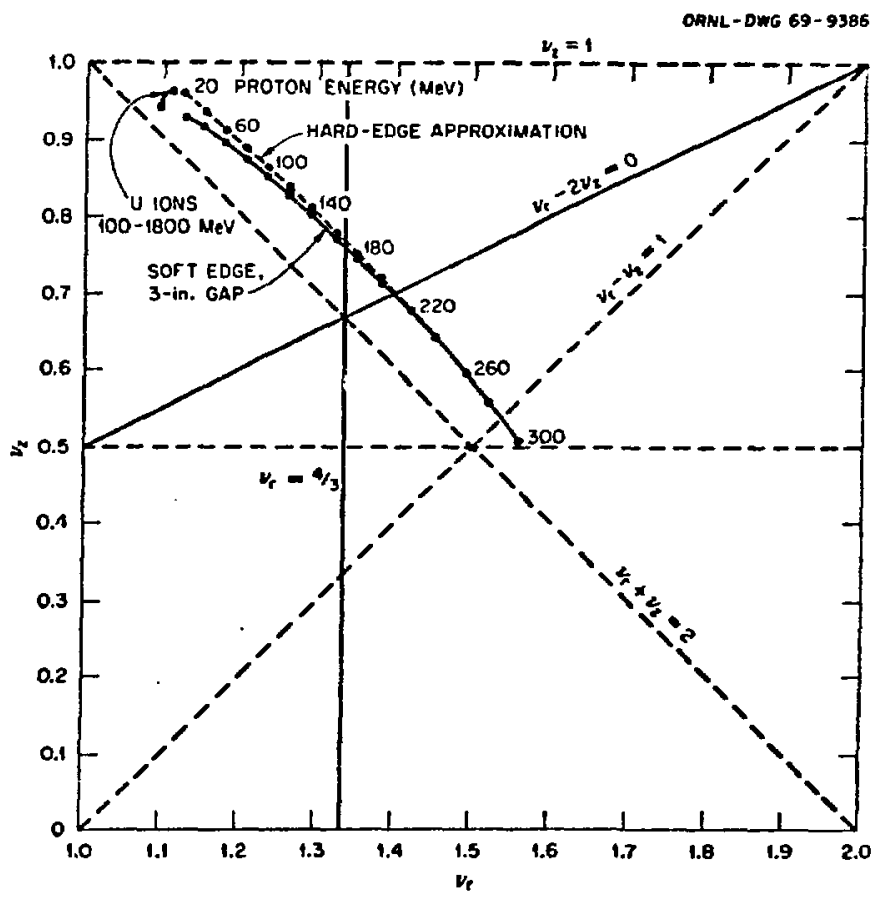

Fig. 1. Radial and axial focusing in the cyclotron.

The sector magnets operate at a maximum flux density of $15 \mathrm{kG}$. Each sector magnet could be provided with 25 trimuing coils varying in width with radius so that adjacent coils span equal numbers of turns. For 300-MeV protons at an energy gain per turn of $1 \mathrm{MeV}$, each coil spans 12 turns. The main characteristics of the magnet are summarized in Table 2 .

1... The concept for the magnet coil system was based on the desire to minimize the encroachment on the RF system space, especially near the center of the cyclotron. The design for the trimming coils follows the general design for the ORIC ${ }^{2}$ except for

the return leads on each coil which encircle the 
Inignet poles adjacent to the main coils in a plane parallel to the nedian piane. The ORIC circular trimming coils are composed of $1 / 4-$ in.-square hollow copper conductor with a $1 / 8-i n$. water passage enclused in vacuum tight stainless steel containers that occupy oniy 1/2-in. of gap. Each coil assembly is insulated by 4 layers of siliconeinpregnated asbestos paper. The triming coils are assumed to be concentric with the bean paths in the magnet. The spacing and radial width of the coils varies from inner to outer radius so as to span equal energy increments (equal numbers of beam turns).

Table 2. Magnet Characteristics

\begin{tabular}{ll}
\hline Number of magnet sectors & 4 \\
Sector angle, degrees & $\Upsilon 47$ \\
Height, ft & 16.5 \\
Diameter, overall, ft & 36.5 \\
Main coil power, kw & 500 \\
Number of trimuing coils & 25 \\
Triming coil polver, kw & 180 \\
Magnet gap, steel, in. & 4 \\
Magnet gap available for & 3 \\
\hline
\end{tabular}

The principal challenges in the design of the magnet are to choose a magnet angle which provides the desired focusing characteristics (taking into account the deviations from the hard-edged approximations for focusing), and trimming coil system that provides smootin adjustment of the radial contour of the meai field from the essentially flat variation characteristic required for heavy ions to the approximately 30\% rise with radius necessary for isochronous acceleration of $300 \mathrm{MeV}$ protons. Preliminary measurements vere made using a $1 / 10$ scale model of a single sector as an aid in developing the design and in preparation for a larger and more exact mode 1 .

\section{Model Measurements}

The model, Fig. 2, was machined from carefully annealed low-carbon steel (SAE 1010, 0.08-0.13\% C). The magnetic Eield measurements were made along the center line of the sector magnet using a high precision $(20.05 \%)$ rotating coil meter. The results of the measurements without trimming coils (Fig. 3) show that the field behaves well up to the 15-kG region, out at higher fields both inner and outer radii begins to sag appreciably. Significant differential saturation occurs at the square edges of the poles. A later design using rounded and stepped pole edge contours preserves the flat field shape at much higher field values than in these studies. 3 ) The nonuniform saturation effects occur at the 17 to $19 \mathrm{kG}$ level are large enough to require consideration in the design of the trimming coil system.

The requirements for trimming coils that adjust the magnetic field for isochronous orbits for all particles were determined experimentally. The model magnet was fitted with 11 trimning coil pairs. The number was limited by the available space in the small magnet. It was necessary to set the model gap at 0.373 in.(equivalent to 4.227 in. full scale),

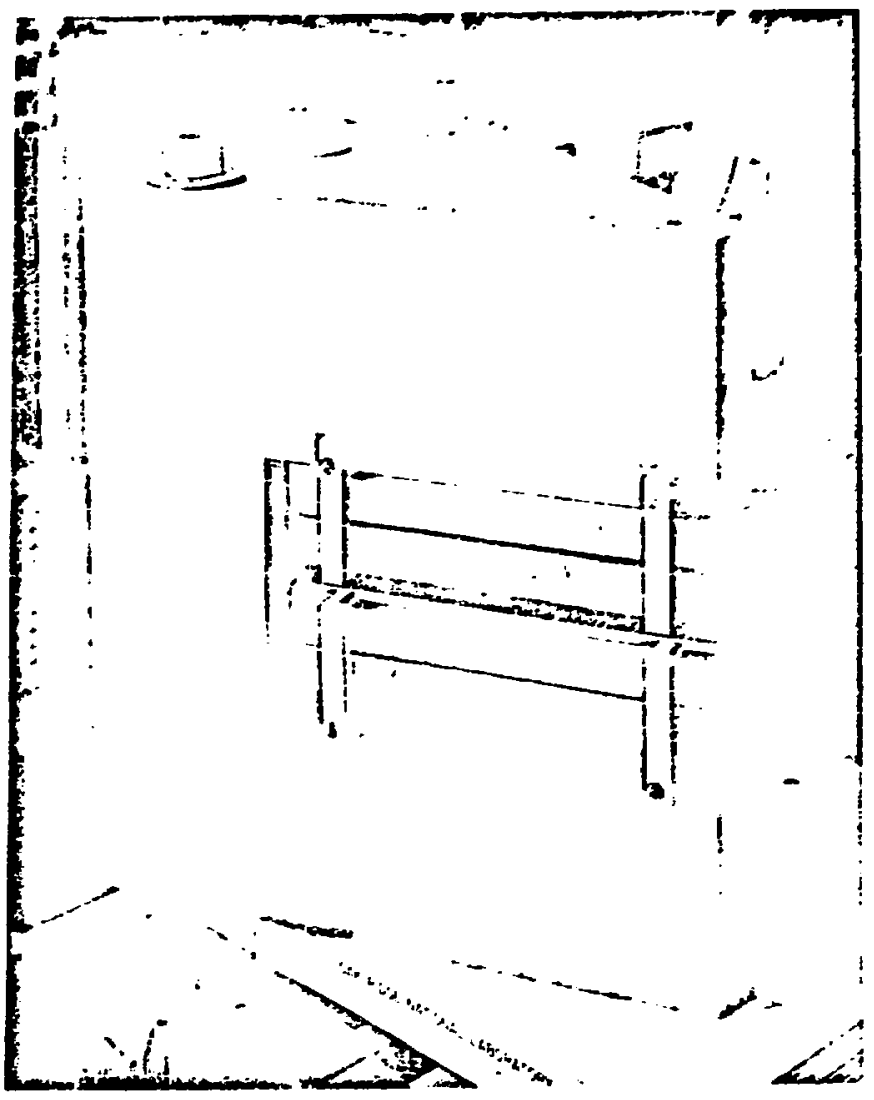

Fig. 2. 1:10-scale model of magnet sector.

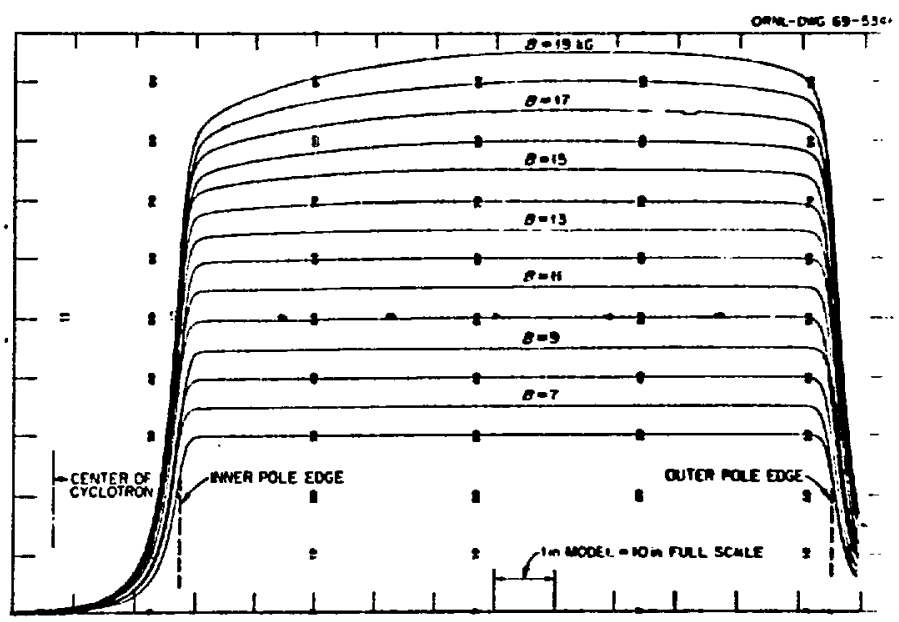

Fig. 3. Magnetic field measurements for magnet model. Data were taken radially in middle of sector.

thereby providing clearance for the 1/4-in.-diamete, magnetic field measuring probe. For this condition there is a $0.020-i n$. air gap in the return yoke. It is suggested that including a similar gap ( 2.25 in. in the full scale magnets would simplify machining and provide a means (by inserting shims) for adjusting the level of the four-sector magnets so that 


\section{their magnotic flelds track.}

The radial widths and positions of the 11 trimming coils were determined by a method developed for the $\mathrm{Mc}^{2}$ cyclotron magnet. ${ }^{4}$ Equal currents in all coils is assumed. The radial width of each trim coil varies inversely with the required radial gradient. Figs, 4 and 5 show the incremental fields of each coil individually at low and high base field respectively. The data are normalized to eliminate the gap, current, and turn number. The field between the poles of an $\mathrm{H}$ - or $\mathrm{C}$-type magnet is given by $B=K_{e} \mu_{0} N I / \ell$, where $I$ is the current, $N$ the number of turns, $l$ the magnet gap length, and $\mu_{0}$ free-space permeability. The factor $k_{e}$, sometimes called magnet efficiency, takes account of the effects of finite permeability of the tips and flux return path. The quantity " $\mathrm{Ke}$ " is plotted in Figs. 4 and 5 , where $K_{e}=B l / \mu_{0} N / I$. Figure 6 shows the base fild and the total field that result as each of the trimming coils is added to the system until all 11 coils are energized at the same current. In Fig. 7, the final results are compared with the magnetic field as isochronized for $300 \mathrm{MeV}$ protons.

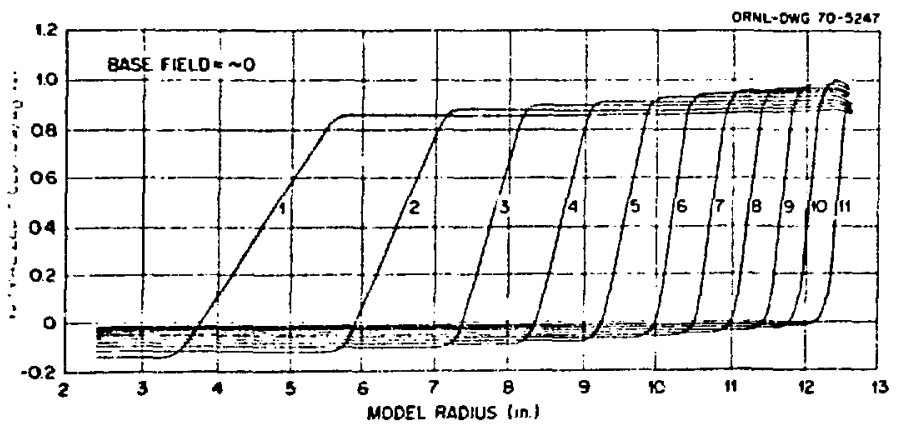

Fig. 4. Magnetic field contribution of each trimming coil at zero base field. Data are normalized. For an infinite permeability yoke all curves would increase from 0 to 1.0 .

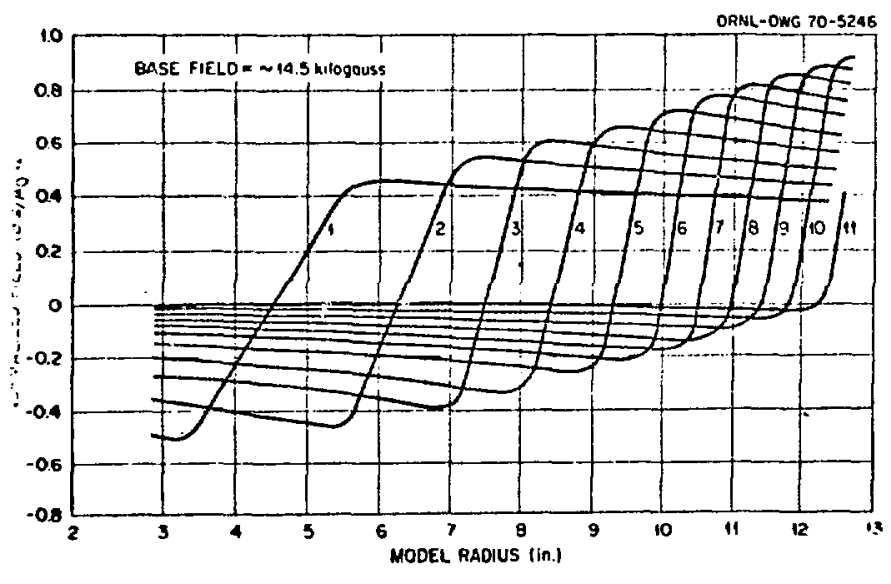

Fig. 5. Magnetic field contribution of each trimming coil at a base field of $14.5 \mathrm{kG}$. The differcnces from $\mathrm{Fig}, 4$ are a result of the nuch lower joke permeability at this flux density.

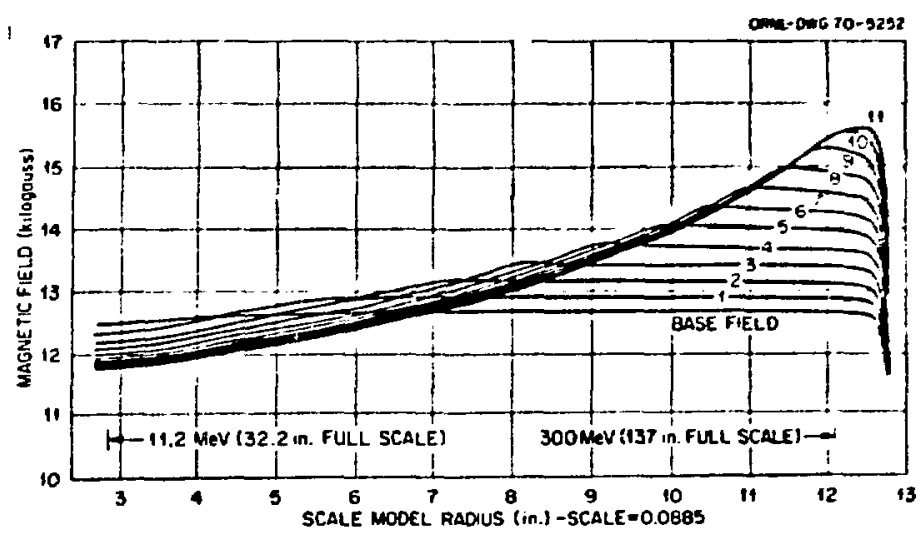

Fig. 6. A tracing of a recording chart to show effect of successive addition of trimming coils. For example, curve 5 is for the base field with first five coils energized.

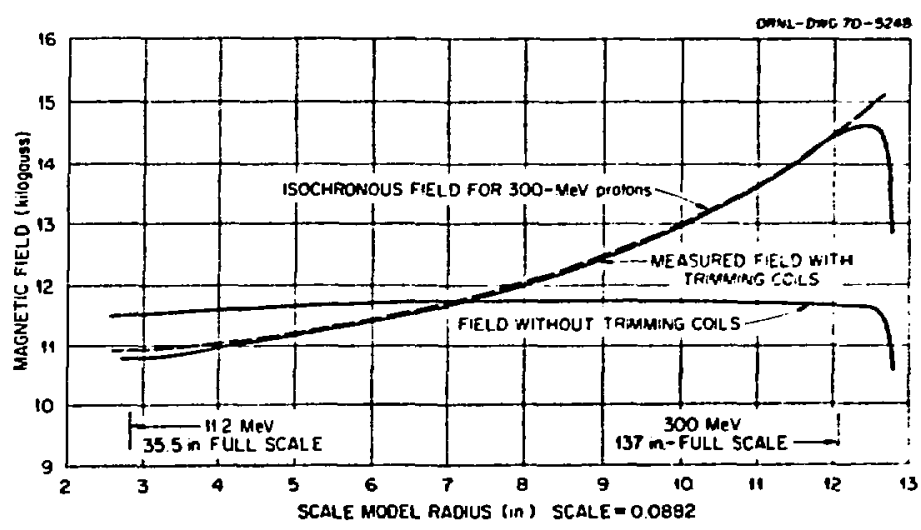

Fig. 7. A comparison of curve 11 of Fig. 6 with the isochronous field for $300-\mathrm{MeV}$ protons.

Radial scans of the field at a base field of 10,12 , and 14 (peak fields of $14,15,15.6$, and $17.3 \mathrm{kG}$ ) are shown in Fig. 8. This demonstrates that saturation effects are small. For fields up to 15.6 peak, the shape remains nearly constant, while at $17.3 \mathrm{kG}$ the shape has changed appreciably near the maximum radius.

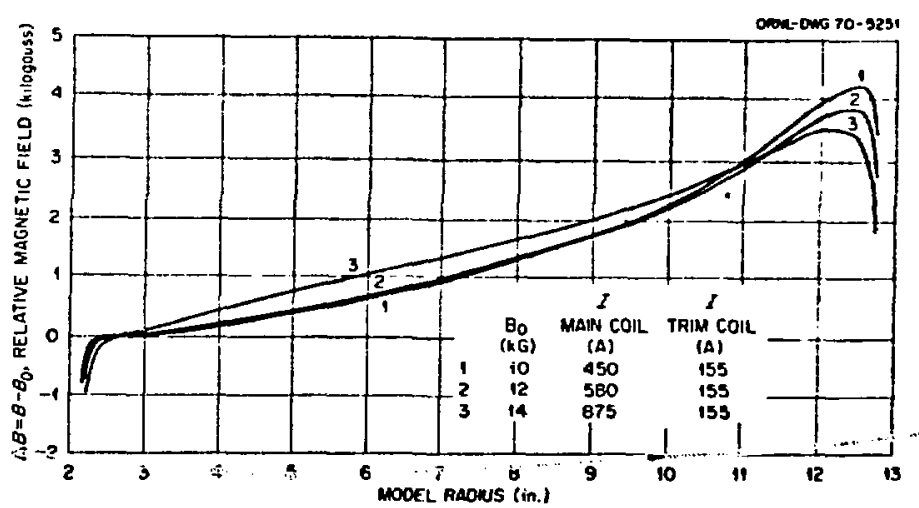

Fig. 8. A comparison of trimming coil contribution at several magnctic field levels to show effects of lower permeability at high fields. 
The test results from the model with its 11 trimming cojls has prompted the consideration of such a system for the full-scale magnet. In Fig. 9 the relative field contribution of the trimning coils is given for $100-$ and $300-\mathrm{McV}$ protons assuming a base field independent of radius. The curves are normalized to unity at maximun radius. The maximum difference between the two curves, only $6.4 \%$, illustrates that a system using one large power supply driving all trimming coils in series, provides over 90\% of the needed correction. Such a system substantially reduces the cost of the triming coil power supplies and the interconnecting cables. A low-current compensating system is needed to supply a few percent of the total correction required for protons, and the small corrections needed to produce an isochronous magnetic field for heavy ions.

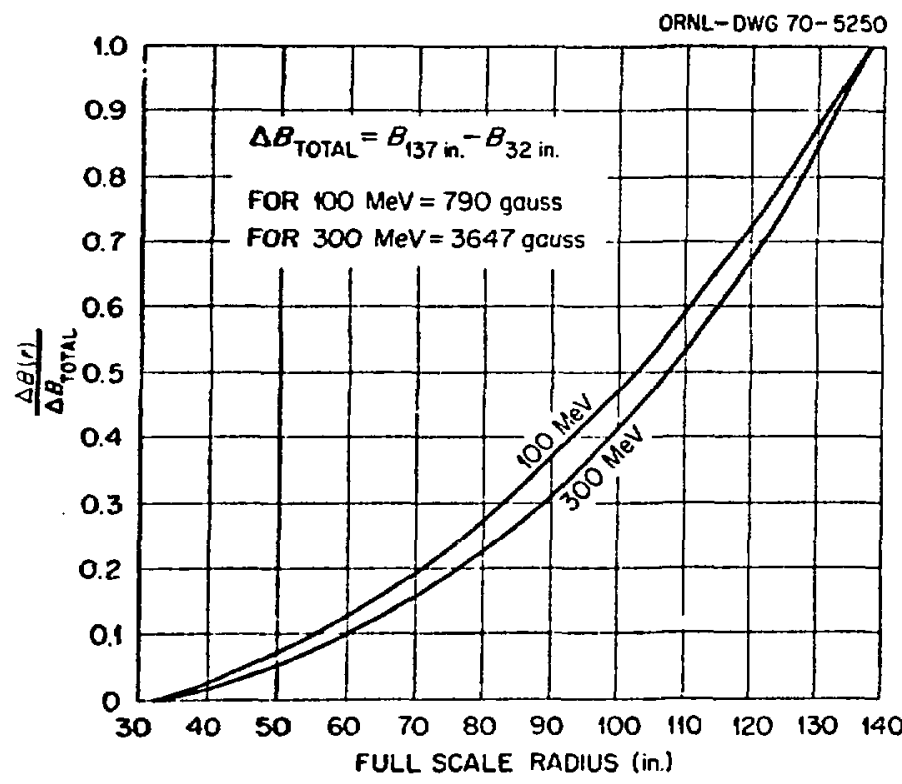

Fig. 9. A comparison of the relative magnetic field corrections for 100 and $300 \mathrm{MeV}$ normalized to 1.0 at maximum radius to show that a trimming coil set with the same value of current in all coils may produce most of the field correction over a wide energy range.

The feasibility of representing the trimming coil magnetic field shapes and the contour of the magnetic field at the pole edge with analytic functions was considered as an aid to further optimization of the design prior to the construction of a larger, more exact model. For the triming coils the function

$$
\begin{aligned}
\Delta B(r)=a_{3} & a_{2} r+a_{3} r^{2} \\
& -\frac{\mu_{0} I}{\pi w} \times \ln \left\{\frac{\cosh \left[\pi\left(r-R_{c}-w / 2\right) / a\right]}{\cosh \left[\pi\left(r-R_{c}+w / 2\right) / a\right]}\right\}
\end{aligned}
$$

was' 'derived to fit the data in Figs. 4 and $5 ; R_{c}$ is the coil radius, $W$ is the coil width, a is the mag net gap, and $I$ is the coil current. The constants $a_{1}$. $a_{2}$, and $a_{3}$ depend on magnet excitation level (yoke flux density) and coil position. At very low fields, the last term, together with $a_{1}=\mu_{0} I / \pi l i$, givos the field distribution quite closely. The last term is exact theoretically for poles of infinite permeability without a return yoke. equation

For the pole-edge fields, it is found that the

$X=0,4+0,7\left[\tanh ^{-1} u+\tanh ^{-1}\left(u^{2}\right)+\tanh ^{-1}\left(u^{3}\right)\right]$,

where $u=1-2 \mathrm{~B} / \mathrm{B}_{\max }$, fits the measurements within approximately 1\% at $15 \mathrm{kG}$ (see Fig. 10). Here, $B_{\max }$ is the value of the magnetic field several gap widths inside the magnet, and $X$ is the distance outward from the magnet edge measured in gap lengths.

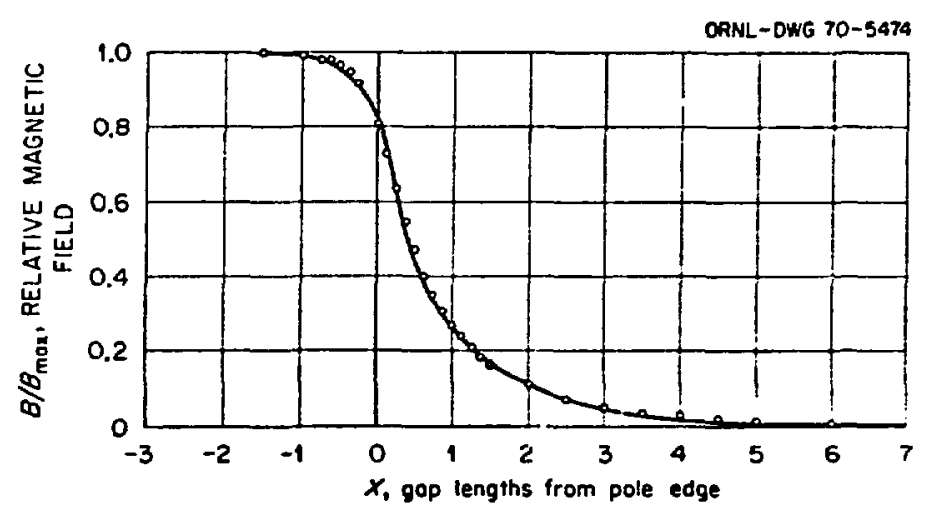

Fig. 10. A comparison of the function $x=0.4+0.7 \sum_{n=1}^{n=3} \tanh ^{-1}\left(u^{n}\right)$

shown as a solid line with the measured data of the field fall-off contour normal to the magnet edge.

As a further aid to design we plan to use computer programs like SYBIL and TRIM, which account for saturation effects. The computed solutions are not exact because of the specialization of the problem to two dimensions, but they are very helpful in the calculation of edge fields and trimming coil fields.

\section{References}

1. J. A. Martin, et al., Fifth International Cyclotron Conference, Butterworths (1971) 41 .

2. E. D. Hudson, et al., Nucl. Instr, and Meth. 18, 19 (1962) 159 .

3. E. D. Hudson, et al., "Magnet Model Studies for Separated-Sector Heavy Ion Cyclotrons," Procecdings of this conference.

4. R. S. Livingston, et al., Nucl. Instr, and Meth. 18, 19 (1962) 438 . 